\title{
BMJ Open Study protocol for a randomised controlled trial of Allen Carr's Easyway programme versus Lambeth and Southwark NHS for smoking cessation
}

Kerry V Wood, ${ }^{1}$ Ian P Albery, ${ }^{1}$ Antony C Moss, ${ }^{1}$ Sarah White, ${ }^{2}$ Daniel Frings ${ }^{1}$

To cite: Wood KV, Albery IP, Moss AC, et al. Study protocol for a randomised controlled trial of Allen Carr's Easyway programme versus Lambeth and Southwark NHS for smoking cessation. BMJ Open 2017;7:e016867. doi:10.1136/ bmjopen-2017-016867

- Prepublication history for this paper is available online. To view please visit the journal online (http://dx.doi.org/10. 1136/bmjopen-2017-016867)

Received 15 March 2017 Revised 20 September 2017 Accepted 2 October 2017

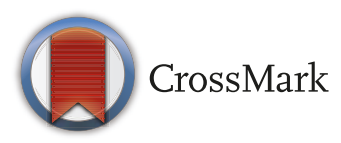

${ }^{1}$ Division of Psychology, London South Bank University, London, UK

${ }^{2}$ Population Health Research Institute, St Georges University of London, London, UK

Correspondence to Dr Kerry V Wood; woodk6@|sbu.ac.uk

\section{ABSTRACT}

Introduction Smoking is a major cause of ill health and is associated with several diseases including cancer, coronary heart disease and stroke. Many psychological and pharmacological smoking cessation treatments are available and although they are undoubtedly the most cost-effective health interventions available, many people still fail to maintain cessation in the longer term. Recently, National Institute for Health and Care Excellence called for comparative studies to determine the short-term and long-term effectiveness of Allen Carr's Easyway (ACE) method of stopping smoking. This study will compare the efficacy of the ACE programme and a 1-1 counselling service available via the National Health Service. Methods and analysis A two-arm, parallel-group, blinded, randomised controlled trial will be conducted with people who smoke tobacco cigarettes, are aged $\geq 18$ years and are motivated to quit. Exclusion criteria comprise self-reported mental health condition, pregnancy or respiratory disease such as chronic obstructive pulmonary disease or emphysema. The primary treatment outcome is smoking cessation 26 weeks after treatment. Participants will be analysed on an intention to treat basis at the point of randomisation. Before being randomised, the research team will not inform participants which two treatments are being compared. Once randomised researchers will be blinded to participant condition, and participants will be blinded to the condition they are not assigned to. Logistic regression will be used to estimate the effectiveness of the treatment condition on smoking cessation at 26 weeks. The following covariates will be included: baseline quit efficacy (at inclusion), age (at inclusion), gender and baseline nicotine dependency.

Ethics and dissemination Approval was granted by LondonFulham Research Ethics Committee (ref: 16/L0/1657). The study's findings will be published in peer-reviewed journals and disseminated at national and international conferences. Trial registration number ClinicalTrials.gov identifier number: NCT02855255. ISRCTN registration number: ISRCTN23584477; Pre-results.

\section{INTRODUCTION}

Dependence on nicotine has long been a problem and although statistics from the $\mathrm{WHO}^{1}$ show that the prevalence of tobacco smoking is declining worldwide, there are still a significant number of people,

\section{Strengths and limitations of this study}

- This study adopted a blinded randomised controlled trial design.

- Chemical verification of quit success.

- Comparison of two common smoking cessation interventions.

- No 'no treatment' control.

- Although researchers are blinded, therapists delivering the interventions are not blinded, that is, each intervention is delivered by experienced facilitators for National Health Service and Allen Carr's Easyway separately.

1.1 billion in 2015 , who continue to smoke. ${ }^{1}$ Smoking is a major cause of ill health and is associated with several diseases including cancer, coronary heart disease and stroke. In the UK alone in 2014, there were 9.6 million smokers, with 78000 deaths attributed to smoking. $^{2}$

Many smokers want to quit and often make several attempts to do so, but the majority fail due to both physiological and psychological factors. ${ }^{3}$ Over the years, researchers have sought to develop effective cessation treatments in an effort to provide education and support. Consequently, many psychological and pharmacological treatments are available to help smokers quit, and although these types of intervention are undoubtedly the most cost-effective interventions available,${ }^{4}$ many people fail to maintain smoking cessation in the longer term. ${ }^{5}$ In 2014, it was reported that $37 \%$ of smokers made an attempt to quit but only $19 \%$ were actually successful. ${ }^{6}$

It is important to understand the relative efficacies of various interventions designed to help people quit, and recently NICE called for comparative studies to determine the short-term and long-term effectiveness of Allen Carr's Easyway (ACE) 
method of stopping smoking. While the ACE method is well established and its efficacy has received some empirical support (see Dijkstra $e t a l^{7}$ ), there has not to date been a randomised control trial (RCT) testing the efficacy of this method. Consequently, ACE method and a 1-1 counselling service available via the NHS will be compared. By comparing the ACE method to a NHS delivered treatment programme, an estimate of the relative effectiveness of ACE in comparison to the NHS service can be made. This will potentially inform future judgements about the use of this method by private and public healthcare providers. The findings will add to the evidence base around the use of the NHS stop smoking service and the ACE method.

\section{METHODS \\ Design}

A two-arm, parallel-group, blinded ${ }^{\mathrm{i}}$, RCT.

\section{Treatment setting/site}

All intervention sessions for the NHS and ACE programmes will be delivered on the London South Bank University (LSBU) Southwark campus and the ACE site in London, SW20. They begin in February 2017 and will run for 8 months.

\section{Study population}

People who smoke tobacco cigarettes and are motivated to quit.

\section{Inclusion and exclusion criteria}

Participants will be eligible provided they are at least 18 years of age, are current smokers who intend to quit and are prepared to be assigned randomly to one of two treatment conditions. Individuals who, on being asked at the point of recruitment, would prefer an NHS-provided treatment, are currently in another RCT or similar research project, disclose that they have a mental health condition, are pregnant or have a respiratory disease such as chronic obstructive pulmonary disease or emphysema will be excluded. Additionally, individuals who feel they are unable to reach the treatment location (LSBU's Southwark Campus or London SW20) for treatment and follow-up will also be excluded.

\section{Interventions}

\section{ACE programme}

The ACE intervention involves a single group session with up to 25 attendees, led by a trained facilitator. The 5-6hour session broadly comprises elements of cognitive behavioural therapy (CBT) and ends with a short hypnotherapy/relaxation exercise. Participants are encouraged to carry on smoking as normal right up until they attend the clinic, and during the session they are encouraged to smoke as normal during scheduled

${ }^{\mathrm{i}}$ With researchers blind to participant condition, and participants blind to the condition they are not assigned to smoking breaks (around every 45-60 min). Participants are assisted in identifying positive expectancies they associate with smoking (eg, pleasure, support, crutch or other benefits) before working towards the conclusion that the belief that smoking provides these benefits is, in fact, erroneous and harmful. Participants also achieve a basic understanding of how the psychological and pharmacological mechanisms of nicotine addiction facilitate the maintenance of erroneous and problematic beliefs. These sessions end with a 'ritual' final cigarette followed by an approximately $20 \mathrm{~min}$ period of hypnotherapy-a light relaxation exercise that reinforces the main points of the session. At the end of each session, each participant is asked to make a written record of what it was about their life as a smoker that made them want to stop. Following each session, the clinical team will call, email or SMS text to the participant on day 1 , and again at $3,7,10,14,21,30,45,60,75$, and 90 days with a short courtesy message such as 'hope you're well. Please don't hesitate to get in touch if you have any questions at all', 'hope all is well with you. Don't forget we're here if you have any questions'.

\section{NHS smoking cessation programme}

The NHS intervention comprises the NHS stop smoking service currently offered at Guy's and St Thomas' NHS Foundation Trust and Lambeth Public Health. This constitutes a single session of around $30 \mathrm{~min}$ which combines motivational interviewing and CBT, followed by four follow-up sessions. The initial session involves informing the client about the treatment programme, assessing current smoking, readiness to quit and past quit attempts. Participants are then advised on the operation of nicotine dependence, the mechanics of withdrawal and given advice on changing routine. The use, pros and cons of various nicotine replacement therapies (NRTs) are also outlined. A quit date is set (within 2 weeks of receiving the intervention) and the importance of complete abstinence discussed. Finally, a carbon monoxide test is administered and explained. Before the session finishes, participants are assisted in identifying high-risk situations in the coming week and develop quit action plans. This treatment is combined with the prescribed NRT of the patients' choice (including Varenicline). One, 2 and 3 weeks post quit date, shorter meetings (approximately $10 \mathrm{~min}$ ) are arranged, respectively. These involve a progress check, a discussion around withdrawal symptoms and coping, a check on NRT supplies, a reflection on difficult situations encountered, a carbon monoxide test and planning for highrisk situations in the coming week. The importance of abstinence is finally reinforced. Four weeks after the quit date, an approximately 10 min meeting checks on progress, measures carbon monoxide, advises on continued use of medication, discusses craving and urges and difficult situations, and finally reinforces the importance of complete abstinence. 


\section{Top-ups and resets}

Both treatments contain, as standard, options to reset/top up quit dates. The ACE provision includes the option for clients to 'top-up' their treatment in two further sessions. These second and third sessions broadly follow the same format (approximately 3.5 hours) but contain more interaction and a smaller group (up to 15 clients) and can be carried out face-to-face or online. They can take place up to 3 months from the initial session. The clinician will provide contact details through which these sessions can be arranged. Should these optional sessions be taken up by a participant then the use of a top-up and the date will be recorded by the clinician on the participant schedule and the subsequent testing dates will be based on the reset date. The primary outcome will be counted from the reset date (eg, prior cigarettes consumed up to that point will be disregarded). The NHS arm contains the option for participants to be given a chance to reset their quit date, at the discretion and suggestion of the clinician. Should a reset be offered, the use of a reset and the revised date will be recorded, and the subsequent testing dates will be based on the reset date. The primary outcome will be counted from the reset date (ie, prior cigarettes consumed up to that point will be disregarded).

Both NHS and ACE treatments offer top-ups that are part of treatment as usual, so it is reasonable that the smoke-free period starts from the date of the top-up (the top-up is part of the normal 'dosage' of the method). Therefore, all participants will have to be smoke-free for 6 months (primary outcome), in line with Russell 6 Standard, from the point they receive the treatment. People who do not slip more than the defined number of times, do not attend top-ups and still maintain from the end of treatment to the final outcome point can of course self-recover. Where participants engage in a subsequent data collection point (ie, 4 weeks) before top-up, these data will be held, but a new set of data will be collected and used in subsequent analysis (see figure 1 for diagram displaying flow of participants through the study).

An alternative approach would be to track all participants from the end of a prespecified 'grace period' which would allow for spontaneous resets. However, such a response would effectively mean that the actual smokefree period between end of treatment and the primary outcome measure point could vary considerably. If there are differences in the number of resets/top-ups used between treatment arms, this would introduce non-trivial systematic bias into the final analysis. In order to explore how the use of top-ups/resets affects outcomes, we will undertake secondary analysis looking only at those who did not use resets/top-ups (and, if appropriate in terms of statistical power, comparing rates between those that do and do not between arms).

\section{Outcomes}

Primary outcome measures

The primary outcome for the trial is the proportion of participants who maintain sustained abstinence for 26 weeks after their quit date. Abstinence is defined using the Russell 6 Standard (ie, fewer than five incidents of smoking from the quit date, including all participants lost to follow-up as failed treatment, and confirming all successful quits via breath carbon monoxide testing). The intention to treat principle will be followed, and those lost to follow-up will be considered as a failed quit. ${ }^{8}$

\section{Secondary outcome measures}

The following secondary outcome measures are being assessed in all participants at 4, 12 and 26 weeks post quit date: self-reported maintenance of smoking cessation: measured by current cessation, number of cigarettes in past week/ month/since last session; use of nicotine replacement therapy/ nicotine containing products: participants will be asked to answer yes/no to the following: 'since we last met, have you regularly used any of the following?' and 'are you planning on using any of the following in the future?' e-cigarettes, nicotine patches, nicotine gum, champix and other; perceived value of being nicotine free: measured on a scale of 1-7 (strongly disagree-strongly agree) to the following items: 'being nicotine free is of value to me', 'I value being nicotine free', 'having no nicotine in my system is/would be beneficial to me'; satisfaction with life $e^{9}$ : a well-validated 5-item scale designed to measure global cognitive judgements of one's life satisfaction. Participants indicate how much they agree or disagree with each of the five items using a scale of 1-7 (strongly disagreestrongly agree); quit efficacy: measured on a scale of 1-7 (strongly disagree-strongly agree) to the following items: 'I can achieve my aims to quit smoking', 'I can cope with the demands of quitting smoking', 'it is unlikely that I will do well at quitting smoking', 'I think I can perform well at quitting smoking'. Readiness to change smoking behaviour ${ }^{10}$ : a well-validated measure of readiness to consider smoking cessation. Measured on a scale of 1 (lowest level of readiness) to 10 (highest level of readiness). Responses 1-3 are indicative of no plans to quit smoking, 4-6 range from thinking about quitting to planning to quit in the next 6 months and $7-10$ range from planning to quit in the next 30 days to having already quit smoking. Adverse events: information regarding any adverse events relating to the participant's health and well-being and whether they are related to treatment.

\section{Sample size}

Tests are powered to detect superiority. Based on data from Dijkstra et $a l^{7}$ an attrition rate between recruitment and final follow-up (at 6 months) of $30 \%$ is indicated. To ensure sufficient participants in the final sample at this rate, an initial sample of 620 participants will be sought (310 per intervention group). To detect differences in success rates (at 6 months) in treatments with success rates of $30 \%$ and $50 \%$, respectively (powered at 0.95 , alpha 0.05 , and adjusting to account for a $25 \%$ loss to follow-up), a sample size of 480 would be required. To detect differences between $20 \%$ and $40 \%$ quit rates (with the same assumptions), a sample of 400 would be 


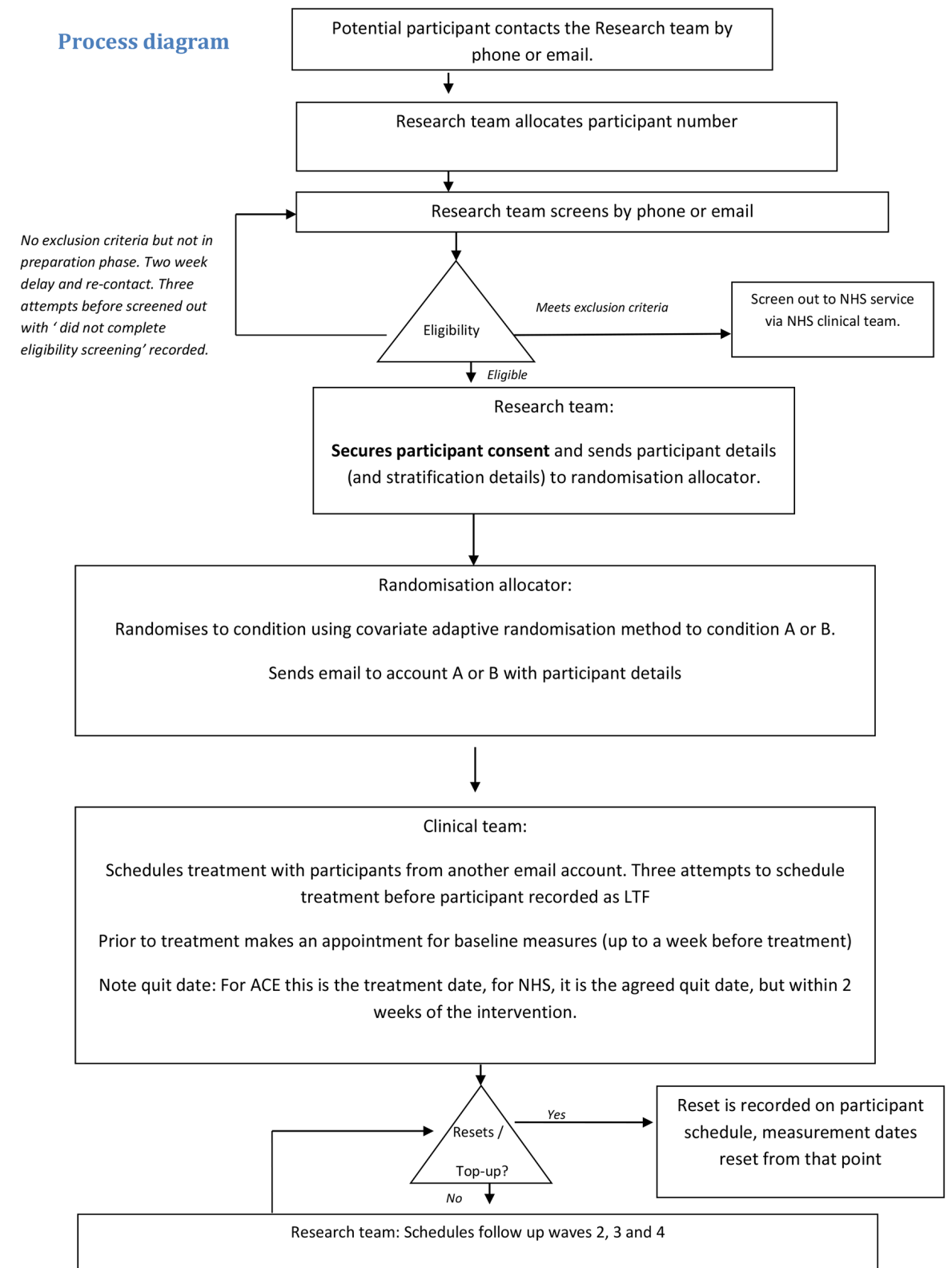

Figure 1 Process diagram indicating the flow of participants through the study. ACE, Allen Carr's Easyway; LTF, lost to followup; NHS, National Health Service.

required. To detect differences between $50 \%$ and $70 \%$, a sample size of 566 is needed. Thus, the study will be adequately powered to detect meaningful differences in the expected range, with lost to follow-up rates in the region of previous research or somewhat above.

\section{Recruitment}

People will be informed of the trial through local placement of posters, leaflets to residential properties, booklets to major employers, employment networks and councils, webpages at LSBU, social media campaigns and radio advertisements. People interested in enrolling will be invited to contact LSBU to provide contact details and will be sent (via email or post) an information sheet containing written information about the study. Within 2 days, potential participants will be contacted by phone and undergo eligibility prescreening. At this point, they will not be informed which two interventions are being compared in the study. If eligible, participants will be asked about demographics, nicotine dependence and prior quit attempts to allow for stratified randomisation into the trial. Within 2 days of being screened, the research team will send eligible participants a consent form (via post or email). As this stage is before intention to treat or condition allocation, no restrictions on contact attempts are placed on this stage. On gaining written consent, participant's details will be sent to the independent randomiser for allocation. Once randomised, participants will see the research team four times (baseline, 4 weeks, 12 weeks and 26 weeks). They will be paid $£ 15$ cash for attending each measurement point and regardless 
of quit outcome, they will be entered into a prize draw to win a Caribbean holiday for two, an iPad or a gym membership.

\section{Randomisation, allocation concealment and sequence generation}

Participants will be randomised to condition by Sarah White (study statistician) using the Kang $e t a l^{11}$ 'Covariate Adaptive Randomization Program' (V.1.0) software package. Four stratification factors, each at two levels, will be used: nicotine dependence (determined by the Fagerstrom Test for Nicotine Dependence (FTND) questionnaire ${ }^{12}$ ), number of prior quit attempts, age and gender. Participants will be assigned to the ACE and NHS 1-1 intervention groups in a ratio of 1:1 (310 in each).

\section{Blinding}

Participants will be blind to both interventions until randomised and once allocated will be blind to the alternative intervention. Members of the trial steering committee, management committee and other team members (with the exception of the statistician/randomiser) will remain blind to treatment allocation until the last follow-up is completed and the data recorded, and the clinical team is not authorised to reveal it.

\section{Data collection, management and analysis}

All data will be collected via paper questionnaires, apart from carbon monoxide readings where a Smokerlyzer piCO analyser will be used. Participant data will be entered by the research team and will only be linked directly with their participant ID code. Personal data (eg, identifiable data) will be accessible to the research team (as part of the screening process), the statistician and the direct care team. Hard copies of data will be destroyed via confidential waste disposal 5 years after the research findings have been published. Electronic copies of data will be stored in two archives. In both cases, only anonymous data will be archived. They will be archived at London South Bank Data Archive and a national data repository such as the UK Data Archive. For more information about data management and monitoring, please see the study research protocol.

\section{Statistical analyses}

A senior statistician determined the sample size and wrote the statistical analysis plan which was subsequently agreed by the steering committee. All statistical analyses will be performed using SPSS V.24 software. Participants will be analysed on an intention to treat basis at the point of randomisation. 95\% CIs will be presented for all analyses. Missing data will be replaced by the mean within condition score. The following stratification variables will be included as covariates in all regression models: baseline quit efficacy (at inclusion), age (at inclusion), gender and baseline nicotine dependency. These covariates have been selected as they have been shown to influence treatment success, and we wish to investigate the unique effects of treatment across a demographically heterogeneous sample.
Primary outcome analysis

Participants for whom smoking cessation cannot be confirmed (ie, are lost to follow-up) will be included in the analysis as failed quits, in line with the Russell 6 Standard. Logistic regression will be used to estimate the effectiveness of the treatment condition on smoking cessation at 6 months.

A series of sensitivity analyses will be conducted to assess the robustness of primary results with regards to definition of the primary outcome. To investigate if the differential effects of interventions are present at each time point ( 4 and 12 weeks), the primary analysis will be repeated twice, the dependent variable being smoking cessation confirmed at 4 and 12 weeks. The primary analysis will be repeated on smoking cessation outcomes at both 12 and 26 weeks using only participants who did not 'reset' their quit dates (NHS arm) or attended a top-up session (ACE arm). This is preferable to including them as failed quits, as many may in fact be successful cessations, while also attending a top-up.

\section{Secondary smoking outcomes}

Use of NRT in each treatment arm will be tested by conducting a logistic regression. Treatment arm and treatment success will be included as the independent variables.

\section{Secondary non-smoking outcomes analyses}

To analyse the take up of treatment between conditions, a logistic regression will be undertaken on treatment completion (operationalised as attendance at the ACE session and attendance at all 5 weeks of the NHS sessions). Multilevel regression models with time of measurement (4 weeks, 12 weeks and 26 weeks) will be undertaken with perceived value of being nicotine free, readiness to change, intentions to re-engage, life satisfaction as the dependent variables and treatment arm as the independent variable. Planned comparisons between treatment arms at each time point will be undertaken.

\section{Plan of presentation}

A CONSORT diagram will be used to describe the sampling, drop-outs and randomisation.

\section{Ethics and Dissemination}

This protocol has been independently peer reviewed by Professor Robert West, University College London and meets requirements of London - Fulham Research Ethics Committee, reference number $16 / \mathrm{LO} / 1657$. In addition, this protocol has been reviewed and approved by the Research Ethics Committee at London South Bank University. Informed consent: Informed written consent will be obtained by the research team. Participants will be sent an information sheet containing information about the study and eligible participants will be required to provide informed written consent before being randomised. At the end of the study, a non-technical summary of the results will be prepared for participants. The study findings will be 
disseminated through national and international conference presentations and will be reported in peer-reviewed journals.

Acknowledgements The authors would like to acknowledge Lambeth Public Health for their support with this study.

Contributors DF, IPA and ACM conceived the design of the trial and secured funding. DF wrote the study protocol. KVW manages the day to day running of the trial; wrote protocol paper with input from all coauthors. SW data analyses. DF is guarantor for this paper. All authors: read and approved the final manuscript.

Funding This trial is funded in full by Allen Carr's Easyway (International) Ltd.

Disclaimer The NHS smoking cessation intervention is provided by Lambeth Stop Smoking Service. The design, conduct,data collection, analyses and interpretation of the trial are conducted by LSBU, independent from Allen Carr's Easy way (International) Ltd. Based on the findings, papers for publication will be prepared by the research team at LSBU who will have ultimate authority over these activities. The research team are contractually free to publish whatever findings the study produces, ACE has no veto over publication, but will be given advanced notice of the findings prior to publication.

Competing interests None declared.

Provenance and peer review Not commissioned; externally peer reviewed.

Open Access This is an Open Access article distributed in accordance with the Creative Commons Attribution Non Commercial (CC BY-NC 4.0) license, which permits others to distribute, remix, adapt, build upon this work non-commercially, and license their derivative works on different terms, provided the original work is properly cited and the use is non-commercial. See: http://creativecommons.org/ licenses/by-nc/4.0/

(C) Article author(s) (or their employer(s) unless otherwise stated in the text of the article) 2017. All rights reserved. No commercial use is permitted unless otherwise expressly granted.

\section{REFERENCES}

1. World Health Organisation. Global report on trends in prevalence of tobacco smoking. $2015 \mathrm{http}: / / w w w . w h o . i n t / t o b a c c o / p u b l i c a t i o n s / s$ urveillance/reportontrendstobaccosmoking/en/

2. Health and Social Care Information Centre. Statistics on smoking. 2016 http://content.digital.nhs.uk/catalogue/PUB20781/stat-smokeng-2016-rep.pdf

3. Buchhalter AR, Acosta MC, Evans SE, et al. Tobacco abstinence symptom suppression: the role played by the smoking-related stimuli that are delivered by denicotinized cigarettes. Addiction 2005;100:550-9.

4. Ronckers ET, Groot W, Ament AJ. Systematic review of economic evaluations of smoking cessation: standardizing the costeffectiveness. Med Decis Making 2005;25:437-48.

5. Stead LF, Perera R, Bullen C, et al. Nicotine replacement therapy for smoking cessation. Cochrane Database Syst Rev 2012;11:CD000146.

6. Public Health England. Health matters: Smoking and quitting in England. 2016 https://www.gov.uk/government/publications/healthmatters-smoking-and-quitting-in-england/smoking-and-quitting-inengland

7. Dijkstra A, Zuidema R, Vos D, et al. The effectiveness of the Allen Carr smoking cessation training in companies tested in a quasiexperimental design. BMC Public Health 2014;14:952.

8. West R, Hajek P, Stead L, et al. Outcome criteria in smoking cessation trials: proposal for a common standard. Addiction 2005;100:299-303.

9. Diener E, Emmons RA, Larsen RJ, et al. The satisfaction with life scale. J Pers Assess 1985;49:71-5.

10. Biener $L$, Abrams DB. The contemplation ladder: validation of a measure of readiness to consider smoking cessation. Health Psychol 1991;10:360-5.

11. Kang M, Ragan BG, Park JH. Issues in outcomes research: an overview of randomization techniques for clinical trials. J Athl Train 2008;43:215-21.

12. Heatherton TF, Kozlowski LT, Frecker RC, et al. The fagerström test for nicotine dependence: a revision of the fagerström tolerance questionnaire. Br J Addict 1991;86:1119-27. 